\title{
Realizing Saturable Absorption and Reverse-saturable Absorption in PEDOT:PSS Film via Electrical Modulation
}

Yanhui Sun,${ }^{\dagger}$ Hui Li,${ }^{\dagger}$ Ruipeng Hou,${ }^{\dagger}$ Mengjuan Diao,${ }^{\dagger}$ Ying Liang, ${ }^{\dagger}$ Zhipeng Huang,

$\dagger^{* *}$ Mark G. Humphrey, ${ }^{\ddagger}$ Chi Zhang ${ }^{\dagger *}$

${ }^{\dagger}$ School of Chemical Science and Engineering, Tongji University, Shanghai, 200092, PR China

${ }^{\star}$ Research School of Chemistry, Australian National University, Canberra, ACT 2601, Australia

${ }^{*}$ Corresponding authors: Z.P. Huang (zphuang@tongji.edu.cn),

C. Zhang (chizhang@tongji.edu.cn)

\section{TOC Graphic}

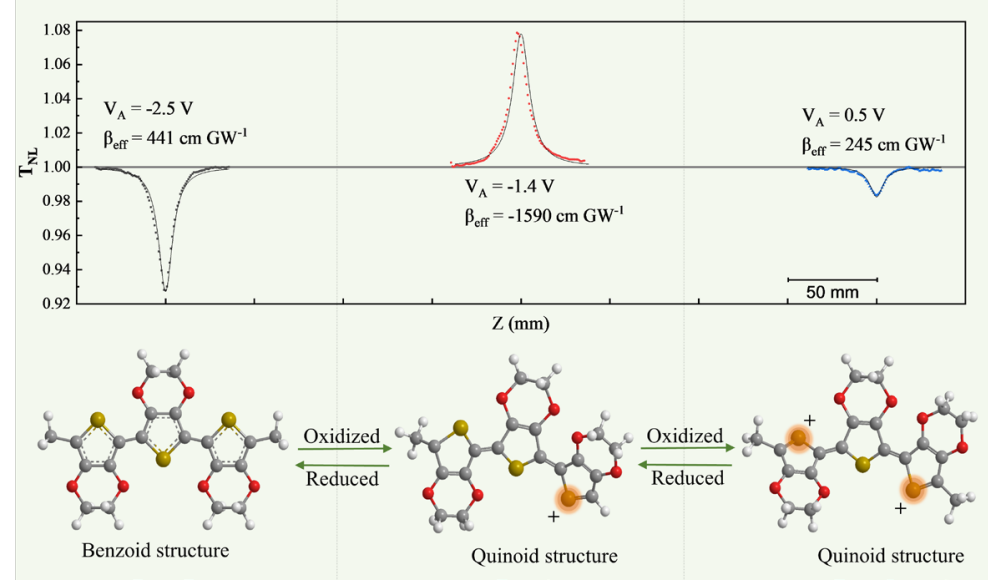

Abstract

The electrical tuning of the nonlinear absorption of materials has promising application potential, while studies remain rare. In this work, we show that the third-order nonlinear absorption of poly(3,4-ethylenedioxythiophene) chemically doped with poly(styrene sulfonic acid) [PEDOT:PSS] can be effectively modulated by external voltage. The 
nonlinear absorption of the film can be varied between reverse saturable absorption (RSA) and saturable absorption (SA) via voltage control with laser excitation at 800 $\mathrm{nm}$, and corresponding nonlinear absorption coefficient can be tuned in the range -1590 to $518 \mathrm{~cm} \mathrm{GW}^{-1}$. The doping level and energy structure of PEDOT are modulated with different voltages. The undoped film affords two-photon absorption and accordingly the RSA response. A moderately doped sample has two polaron levels, and Pauli blocking associated with these two polaron levels results in SA. The bipolaron level in heavily doped PEDOT leads to excited state absorption and therefore RSA behavior. The approach reported here can be applied to other semiconductors, being a convenient, effective, and promising method for the electrical tuning of the optical nonlinearity.

\section{Keyword}

PEDOT, conductive polymer, nonlinear optical, electrical control, polaron

\section{Introduction}

Dynamical modulation of materials' property, especially via electric stimulus, is the fundamental principle of a variety of application, including data storage, signal process, display, communication, etc. The electrical modulating of optical nonlinearity is attracting intensive attention, due to the wide application range of laser. ${ }^{1,2}$ Study concerning the electrical modulation of optical nonlinearity can be traced back several decades, but a high electrical field (kilovolts/cm) is necessary to induce noticeable modulation. ${ }^{3}$ The electrical field has been remarkably reduced recently because 
advanced nano-machining enables fine control over the size and arrangement of active materials. The nonlinear optical (NLO) responses have been electrically modulated in a series of nanostructure, including the plasmonic structures of gold, ${ }^{4-7}$ graphene, ${ }^{8}$ and silicon, ${ }^{9}$ as well as two-dimensional layered structures $\left(\mathrm{WSe}_{2},{ }^{10} \mathrm{MoTe},{ }^{11} \mathrm{MoS}_{2},{ }^{12}\right.$ graphene $\left.{ }^{13}\right)$, CdS nanobelt, ${ }^{14}$ organic conjugated polymer, ${ }^{15}$ etc. However, most efforts have been devoted to modulate the frequency conversion of laser, which is a parametric NLO response.

In a nonparametric NLO process, the transfer of electrons from one real level to another level occurs, and nonlinear absorption is a typical nonparametric NLO process that has extensive application. ${ }^{16-19}$ The modulation of nonlinear absorption by external stimuli has been demonstrated. One of us has modulated the nonlinear absorption properties of a series of Ru-based molecular inorganic compounds by electrochemical treatment. ${ }^{20-23}$ Hou and Song et al. have tuned the nonlinear absorption of Rhodamine B salicylaldehyde hydrazone metal complex by UV irradiation. ${ }^{24}$ However, these studies focused on molecules dispersed in solution. The dynamical tuning of the nonlinear absorption of a solid film is highly desirable for on-chip optoelectrical application, while it has not been reported so far.

In this work, the electrical modulation of the nonlinear absorption of a thin film of poly(3,4-ethylenedioxythiophene) chemically doped with poly(styrene sulfonic acid) [PEDOT:PSS] is studied. PEDOT:PSS is a conductive polymer becoming a subject of intense interest (e.g. organic electrochemical transistors ${ }^{25}$, electrochromic devices ${ }^{26}$, neural electrodes ${ }^{27}$, surface switch devices ${ }^{28}$, electronic plants, ${ }^{29}$ etc.), because it can be 
coupled with ionic and electronic charge species to control the electronic, physical, and chemical properties of material. ${ }^{30}$ We show here that the nonlinear absorption of the PEDOT:PSS film can be varied between reverse saturable absorption (RSA) and saturable absorption (SA) via voltage control with laser excitation at $800 \mathrm{~nm}$, and corresponding nonlinear absorption coefficient ( $\left.\beta_{\text {eff }}\right)$ can be tuned in the range -1590 to $518 \mathrm{~cm} \mathrm{GW}^{-1}$. An electrochemical process occurs during the variation of applied voltage $\left(V_{A}\right)$, resulting in different doping states of PEDOT:PSS (undoped, moderately doped, and heavily doped). The RSA is ascribed to two-photon absorption (TPA) in the undoped state and excited-state absorption (ESA) in the heavily doped state, and the SA is ascribed to Pauli blocking in the moderately doped PEDOT:PSS. The approach introduced here is convenient and effective, and can be applied to the electrical tuning of the NLO response of other materials. 


\section{Results and Discussion}

\subsection{Device Structure}

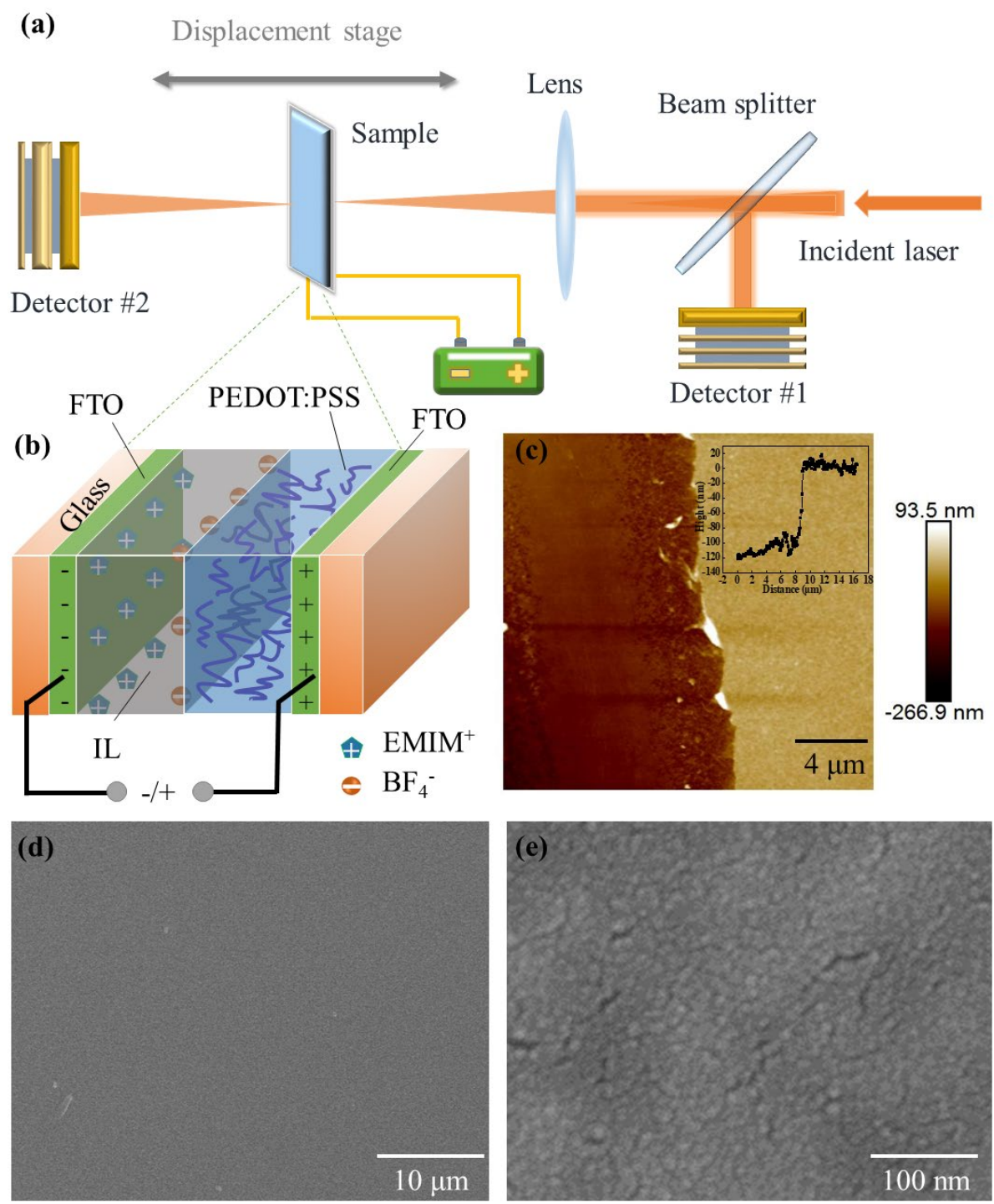

Figure 1. (a) Schematic of an open-aperture Z-scan setup. (b) Schematic structure of PEDOT:PSS device. (c) AFM image of PEDOT:PSS film and corresponding height profile. (d, e) SEM images of PEDOT:PSS film.

The NLO response of the PEDOT:PSS film is modulated via electrochemical treatment, basing on the fact that electrochemical treatment will vary the electronic structure of the PEDOT:PSS. The experimental setup is illustrated by Figure 1a, composing an open-aperture Z-scan setup and a power supply applying a desirable 
voltage to the PEDOT:PSS film. The PEDOT:PSS loaded onto a fluorine-doped tin oxide (FTO) substrate acts as a working electrode, and was assembled into a miniature electrochemical cell, with another FTO substrate serving as a counter electrode, and ionic liquid (IL) as an electrolyte. The IL used is 1-ethyl-3-methylimidazolium tetrafluoroborate (EMIM BF $)$. A Surlyn ${ }^{\circledR}$ membrane $(60 \mu$ m thick) was used to space and bind two electrodes. A typical digital camera image of a device for the Z-scan measurement is shown in Figure S1 in the supporting information (SI). The charging state of the PEDOT:PSS film under positive voltage is schematically illustrated in Figure $1 \mathrm{~b}$. When voltage is applied the energy levels and/or bands of PEDOT will be varied due to the reduction or oxidation of PEDOT. Meanwhile, the occupation of these energy levels and/or bands will be also modified. The modification of energy levels/bands and their occupation will initiate different NLO responses, resulting in the electrochemical modulation of the NLO response of the PEDOT:PSS film.

Atomic force microscopy (AFM) was used to characterize the quality and thickness of PEDOT:PSS film. Figure 1c and Figure S2 in SI show that the PEDOT:PSS film is smooth and homogeneous. A cross-sectional height profile near the scratch edge (inset of Figure 1c) indicates that the thickness of the film is $c a 120 \mathrm{~nm}$. Scanning electron microscopy (SEM) images confirm the homogeneity f PEDOT:PSS films (Figure 1d, and 1e). 


\subsection{Electrical Modulation of the NLO Response}

(a)

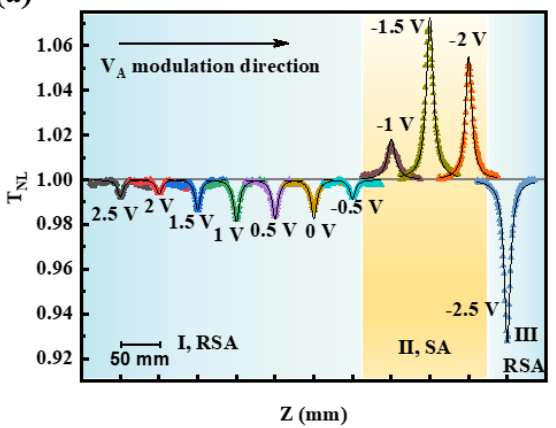

(b)

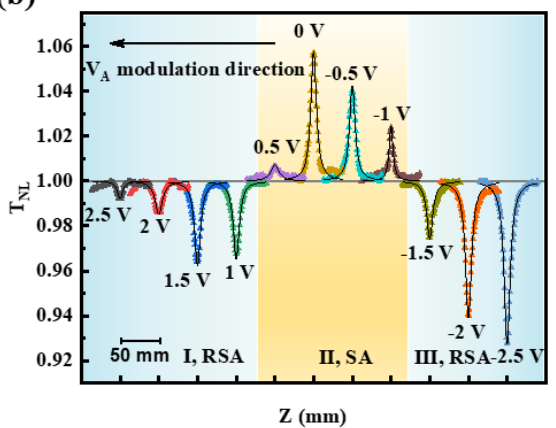

(c)

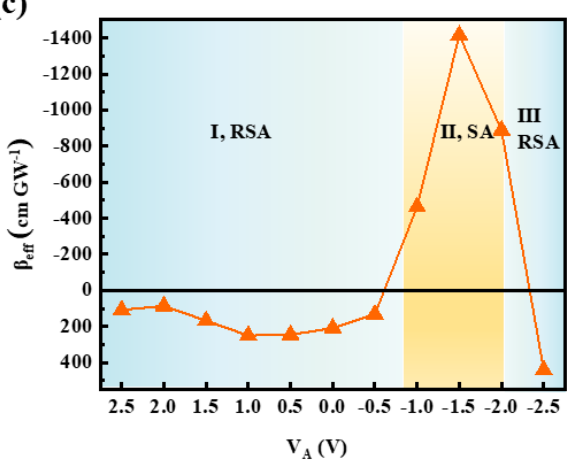

(d)

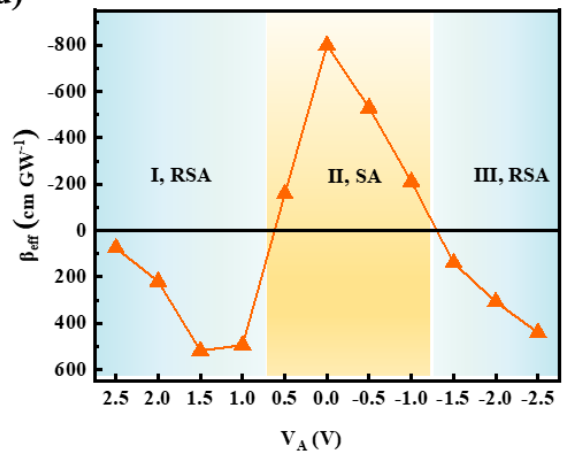

Figure 2. Z-scan curves of device at $800 \mathrm{~nm}$ under $\mathrm{V}_{\mathrm{A}}$ varying (a) from $2.5 \mathrm{~V}$ to -2.5 $\mathrm{V}$ and (b) from $-2.5 \mathrm{~V}$ to $2.5 \mathrm{~V}$. The $\beta_{\text {eff }}$ values of the devices under $\mathrm{V}_{\mathrm{A}}$ varying (c) from 2.5 to $-2.5 \mathrm{~V}$ and (d) from -2.5 to $2.5 \mathrm{~V}$.

The electrical modulation of the NLO response of devices was investigated by an open-aperture Z-scan setup. The voltage was applied to the cell for a sufficiently long time to achieve an equilibrium charging state (i.e. stable linear transmittance), and then Z-scan was carried out with the same $\mathrm{V}_{\mathrm{A}}$. A series of $\mathrm{V}_{\mathrm{A}}$ were applied to the cell, with forward $\mathrm{V}_{\mathrm{A}}$ modulation referencing to decrease $\mathrm{V}_{\mathrm{A}}$ and backward $\mathrm{V}_{\mathrm{A}}$ modulation to increase $\mathrm{V}_{\mathrm{A}}$. The $\mathrm{V}_{\mathrm{A}}$ was varied in the range $-2.5 \mathrm{~V}$ to $2.5 \mathrm{~V}$ to avoid possible degradation of the IL. In each Z-scan experiment, the normalized transmittance $\left(\mathrm{T}_{\mathrm{NL}}\right)$ of the device was measured as a function of distance to the focal point of the lens $(Z)$.

The corresponding $\mathrm{T}_{\mathrm{NL}}(\mathrm{Z})$ curves are plotted in Figure $2 \mathrm{a}$ and $2 \mathrm{~b}$, which show that the NLO response of the PEDOT:PSS film can be controllably modified via $\mathrm{V}_{\mathrm{A}}$. In both 
forward (Figure $2 \mathrm{a}$ ) and backward $\mathrm{V}_{\mathrm{A}}$ modulation (Figure $2 \mathrm{~b}$ ), three $\mathrm{V}_{\mathrm{A}}$ regions can be defined according to the NLO response of PEDOT:PSS. For forward $\mathrm{V}_{\mathrm{A}}$ modulation The PEDOT:PSS film shows RSA response with $\mathrm{V}_{\mathrm{A}}$ in the range 2.5 to $-0.5 \mathrm{~V}$ (Region I), in which the $\mathrm{T}_{\mathrm{NL}}(\mathrm{Z})$ decreases with sample closer to the focus of laser (i.e. larger laser intensity). The PEDOT:PSS film exhibits SA behavior in $\mathrm{V}_{\mathrm{A}}$ region II (-1 to $\left.-2 \mathrm{~V}\right)$. In this case, the $\mathrm{T}_{\mathrm{NL}}(\mathrm{Z})$ increases as the sample closer to the focus. In region III (-2.5 V) the NLO response of PEDOT transforms to RSA. It will be discussed below that different NLO responses are associated with different structures and doping features of PEDOT in the film. In backward $\mathrm{V}_{\mathrm{A}}$ modulation, there are also three regions (Figure $2 b$ ), while the $\mathrm{V}_{\mathrm{A}}$ range is different from those in forward $\mathrm{V}_{\mathrm{A}}$ modulation. As shown in the Figure $\mathrm{S} 3$ in the SI, the variation of $\mathrm{T}_{\mathrm{NL}}(\mathrm{Z})$ of a bare device (a device assembled by two bare FTO substrates and injected with IL) with different $\mathrm{V}_{\mathrm{A}}$ is negligible in comparison with the PEDOT:PSS device, confirming that the variation of optical nonlinearity comes exclusively from the $\mathrm{V}_{\mathrm{A}}$ modulation of PEDOT:PSS.

The effective third-order nonlinear absorption coefficient $\left(\beta_{\text {eff }}\right)$ was derived from $\mathrm{T}_{\mathrm{NL}}(\mathrm{Z})$ curves via data fitting (Experimental section). The results are shown in Figure $2 \mathrm{c}$ for forward $\mathrm{V}_{\mathrm{A}}$ modulation and Figure $2 \mathrm{~d}$ for backward $\mathrm{V}_{\mathrm{A}}$ modulation. The $\beta_{\text {eff }}$ can be monotonically modulated via $\mathrm{V}_{\mathrm{A}}$ between the maximum and the minimum. For forward $V_{A}$ modulation, the $\beta_{\text {eff }}$ firstly increases from 100 to $300 \mathrm{~cm} \mathrm{GW}^{-1}$ with decreasing $\mathrm{V}_{\mathrm{A}}$ to $1 \mathrm{~V}$, and then $\beta_{\text {eff }}$ monotonically decreases to $-1415 \mathrm{~cm} \mathrm{GW}^{-1}$ with further $\mathrm{V}_{\mathrm{A}}$ decreasing to $-1.5 \mathrm{~V}$. Beyond this value the $\beta_{\mathrm{eff}}$ increases with decreasing $\mathrm{V}_{\mathrm{A}}$. The backward $\mathrm{V}_{\mathrm{A}}$ modulation shows a similar feature. The close correlation between 
$\beta_{\text {eff }}$ and $\mathrm{V}_{\mathrm{A}}$ enables the on-demand modulation of the NLO performance of the PEDOT. The largest value of $\beta_{\text {eff }}$ from modulated PEDOT:PSS film is $-1590 \mathrm{~cm} \mathrm{GW}^{-1}$ achieved with a $\mathrm{V}_{\mathrm{A}}$ of $-1.4 \mathrm{~V}$ (Figure $\mathrm{S} 4 \mathrm{a}$ in the $\mathrm{SI}$ ). The $\beta_{\text {eff }}$ of typical semiconductor materials showing SA response are listed in Table 1. It is shown that the maximum value of the $\beta_{\text {eff }}$ of PEDOT:PSS is larger than those of most semiconductors with laser excitation at $800 \mathrm{~nm}$ wavelength. Meanwhile, the Z-scan experiments were carried out with the $\mathrm{V}_{\mathrm{A}}$ repeatedly varied between $-1.4 \mathrm{~V}$ and $1 \mathrm{~V}$ (Figure S4b in the SI), demonstrating that the modulation of $\beta_{\mathrm{eff}}$ is reversible.

Table 1. NLO parameters of typical semiconductors

\begin{tabular}{|c|c|c|c|}
\hline Materials & $\lambda(\mathbf{n m})$ & $\beta_{\text {eff }}\left(\mathrm{cm} \mathrm{GW} \mathbf{G W}^{-1}\right)$ & Type of NLO \\
\hline $\mathrm{ITO}^{31}$ & 1240 & $\sim-7500$ & $\mathbf{S A}$ \\
\hline $\mathrm{Cu}_{2-\mathrm{x}} \mathrm{S}^{32}$ & 1300 & -161 & SA \\
\hline ITO-5 $5^{33}$ & 1500 & -51.40 & SA \\
\hline ITO- $12^{33}$ & 1300 & -47.03 & SA \\
\hline $\mathrm{Bi}_{2} \mathrm{O}_{2} \mathrm{Se}^{34}$ & 800 & -2910 & SA \\
\hline $\mathrm{Bi}_{2} \mathrm{O}_{2} \mathrm{Se}^{34}$ & 1500 & -648 & SA \\
\hline $\mathrm{CuS}^{35}$ & 1030 & $-1.015 \pm 0.007$ & SA \\
\hline $\mathrm{WS}_{2}{ }^{36}$ & 800 & $-397 \pm 40$ & SA \\
\hline Black Phosphorus ${ }^{37}$ & 800 & -0.0138 & SA \\
\hline $\mathrm{MoS}_{2}{ }^{38}$ & 800 & $-(4.60 \pm 0.27) \times 10^{-3}$ & SA \\
\hline graphene $^{39}$ & 800 & -20 & SA \\
\hline graphene oxide $^{39}$ & 800 & -40 & SA \\
\hline $\mathrm{AgInSe}_{2}$ nanorods $^{40}$ & 800 & -0.29 & SA \\
\hline Au nanorods ${ }^{41}$ & 800 & -1.5 & SA \\
\hline PEDOT:PSS (this work) & 800 & -1590 & SA \\
\hline
\end{tabular}


The PEDOT shows hysteresis in $\beta_{\text {eff }}-V_{A}$ characteristics when the $V_{A}$ was swept in sequential forward and backward $\mathrm{V}_{\mathrm{A}}$ modulation. This is a typical hysteresis effect occurring in electric double layer charged transistor or liquid gate transistor, which arises from the slow electrochemical process PEDOT associated with $\mathrm{V}_{\mathrm{A}}$ modulation. ${ }^{42}$

\subsection{Process during Electrical Modulation}

(a)

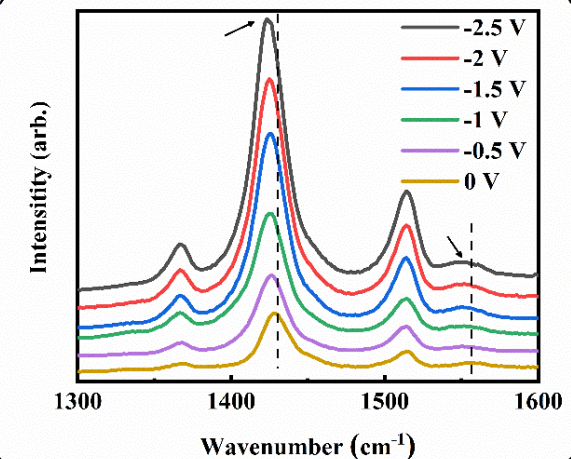

(c)

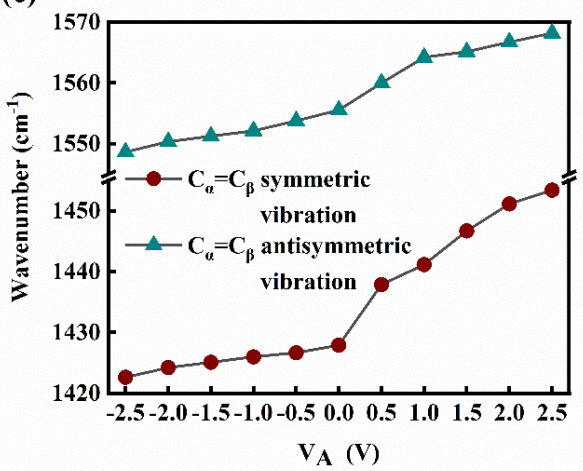

(b)

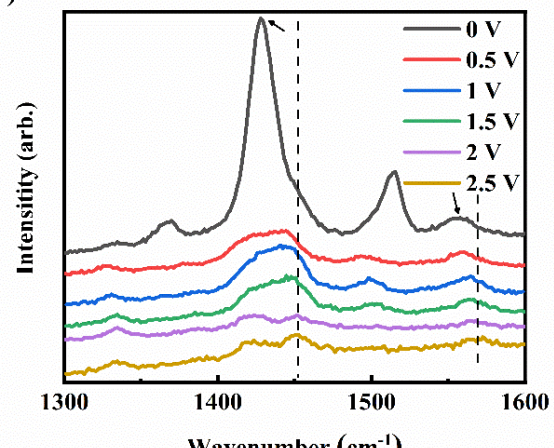

(d)

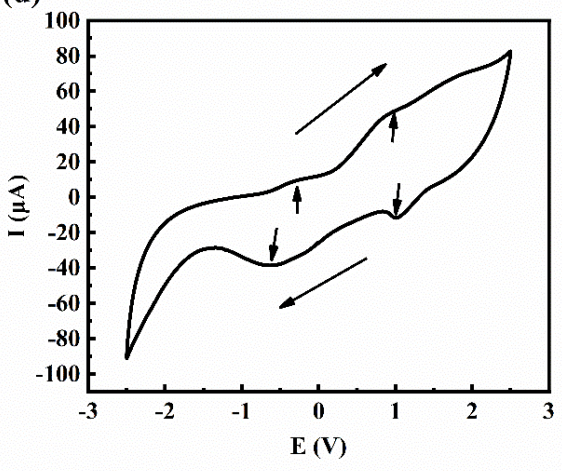

Figure 3. (a,b) In-situ Raman spectra of PEDOT:PSS film for $\mathrm{V}_{\mathrm{A}}$ varying in the range -2.5 to $2.5 \mathrm{~V}$. (c) The shift of Raman peaks with $\mathrm{V}_{\mathrm{A}}$. (d) CV of PEDOT:PSS.

The $\beta_{\text {eff- }}-V_{A}$ characteristic of PEDOT:PSS film should be associated with the modulation of the electron state PEDOT, and both electrostatic doping or electrochemical doping might possibly modulate the electron state of PEDOT. In electrostatic doping, an electric double layer forming at the interface of dielectrics and solid functions as a nanoscale capacitor that charges and/or discharges the solid via a non-faradaic process. Differently, the charging and discharging are accomplished by 
the oxidation and reduction of material during electrochemical doping. The in-situ Raman experiment, cyclic voltammetry (CV), and Vis-NIR spectroscopy suggest that the $\mathrm{V}_{\mathrm{A}}$-related NLO response of the PEDOT:PSS film arises from the electrochemical doping of the PEDOT.

The structural variation of PEDOT under different $\mathrm{V}_{\mathrm{A}}$ is indicated by in-situ Raman spectra. The in-situ Raman spectra are shown in Figure 3a and Figure $3 \mathrm{~b}$ for backward $\mathrm{V}_{\mathrm{A}}$ modulation, and the spectra are plotted in the wavenumber range of 1300 to $1600 \mathrm{~cm}^{-1}$ to show clearly the shift of Raman peaks. When a $\mathrm{V}_{\mathrm{A}}$ of $-2.5 \mathrm{~V}$ was applied, $\mathrm{C}_{\alpha}=\mathrm{C}_{\beta}$ antisymmetric vibrations and $\mathrm{C}_{\alpha}=\mathrm{C}_{\beta}$ symmetric vibrations were located at 1548 $\mathrm{cm}^{-1}$ and $1422 \mathrm{~cm}^{-1},{ }^{43}$ respectively. With the $\mathrm{V}_{\mathrm{A}}$ gradual increasing, both $\mathrm{C}_{\alpha}=\mathrm{C}_{\beta}$ antisymmetric vibrations and $\mathrm{C}_{\alpha}=\mathrm{C}_{\beta}$ symmetric vibrations were shifted toward larger wavenumber. Finally, the $\mathrm{C}_{\alpha}=\mathrm{C}_{\beta}$ antisymmetric vibrations and $\mathrm{C}_{\alpha}=\mathrm{C}_{\beta}$ symmetric vibrations were shifted to 1568 and $1453 \mathrm{~cm}^{-1}$ for a $\mathrm{V}_{\mathrm{A}}$ of $2.5 \mathrm{~V}$. Meanwhile, the peaks corresponding to positive $\mathrm{V}_{\mathrm{A}}$ are much weaker than those corresponding to negative $\mathrm{V}_{\mathrm{A}}$. This is associated with the electronic screen of photons resulting from the high conductivity of positive charged PEDOT. ${ }^{44}$ The detailed variation of the $\mathrm{C}_{\alpha}=\mathrm{C}_{\beta}$ antisymmetric vibrations and $\mathrm{C}_{\alpha}=\mathrm{C}_{\beta}$ symmetric vibrations are plotted in Figure $3 \mathrm{c}$, showing clearly the linear variation of the Raman peaks with the $\mathrm{V}_{\mathrm{A}}$. The in-situ Raman spectra in backward $\mathrm{V}_{\mathrm{A}}$ modulation (Figure $\mathrm{S} 5$ in the $\mathrm{SI}$ ) is identical to that in forward modulation. The variation of Raman peaks evidences that the structure of PEDOT was modified due to external $\mathrm{V}_{\mathrm{A}}$. These changes are consistent with the transformation of 
the benzoid structure into quinoid one upon doping, because in this case the $\mathrm{C}_{\alpha}=\mathrm{C}_{\beta}$ force constant will decrease. ${ }^{45}$

$\mathrm{CV}$ experiment confirms the redox process of the PEDOT. Figure $3 \mathrm{~d}$ shows the cyclic voltammograms of the PEDOT:PSS film. The cyclic voltammograms of IL on a bare device (Figure S6a in the SI) show that no apparent redox peak can be found, indicating that the redox peaks found in Figure $3 \mathrm{~d}$ are associated with the oxidation and reduction of the PEDOT. The oxidation peak near a $\mathrm{V}_{\mathrm{A}}$ of $-0.3 \mathrm{~V}$ and the reduction peak near a $\mathrm{V}_{\mathrm{A}}$ of $-0.7 \mathrm{~V}$ suggest that the redox process occurs during $\mathrm{V}_{\mathrm{A}}$ variation. The oxidation peak near a $\mathrm{V}_{\mathrm{A}}$ of $1.1 \mathrm{~V}$ and the reduction peak near a $\mathrm{V}_{\mathrm{A}}$ of $1 \mathrm{~V}$ suggest another redox process. In the oxidation cycle, the first peak $(-0.3 \mathrm{~V})$ corresponds to the removal of the first electron from the polymer, and thus to the generation of polarondominated mid-gap states. ${ }^{46}$ The second peak $(1.1 \mathrm{~V})$ corresponds to the removal of the second electron with the generation of bipolaron-dominated states. In the oxidation cycle, two peaks also correspond to bipolaron and polaron state, respectively. The repeated cyclic voltammograms of the PEDOT (Figure S6b in the SI) show that the oxidation and reduction of the PEDOT is highly reversible. 

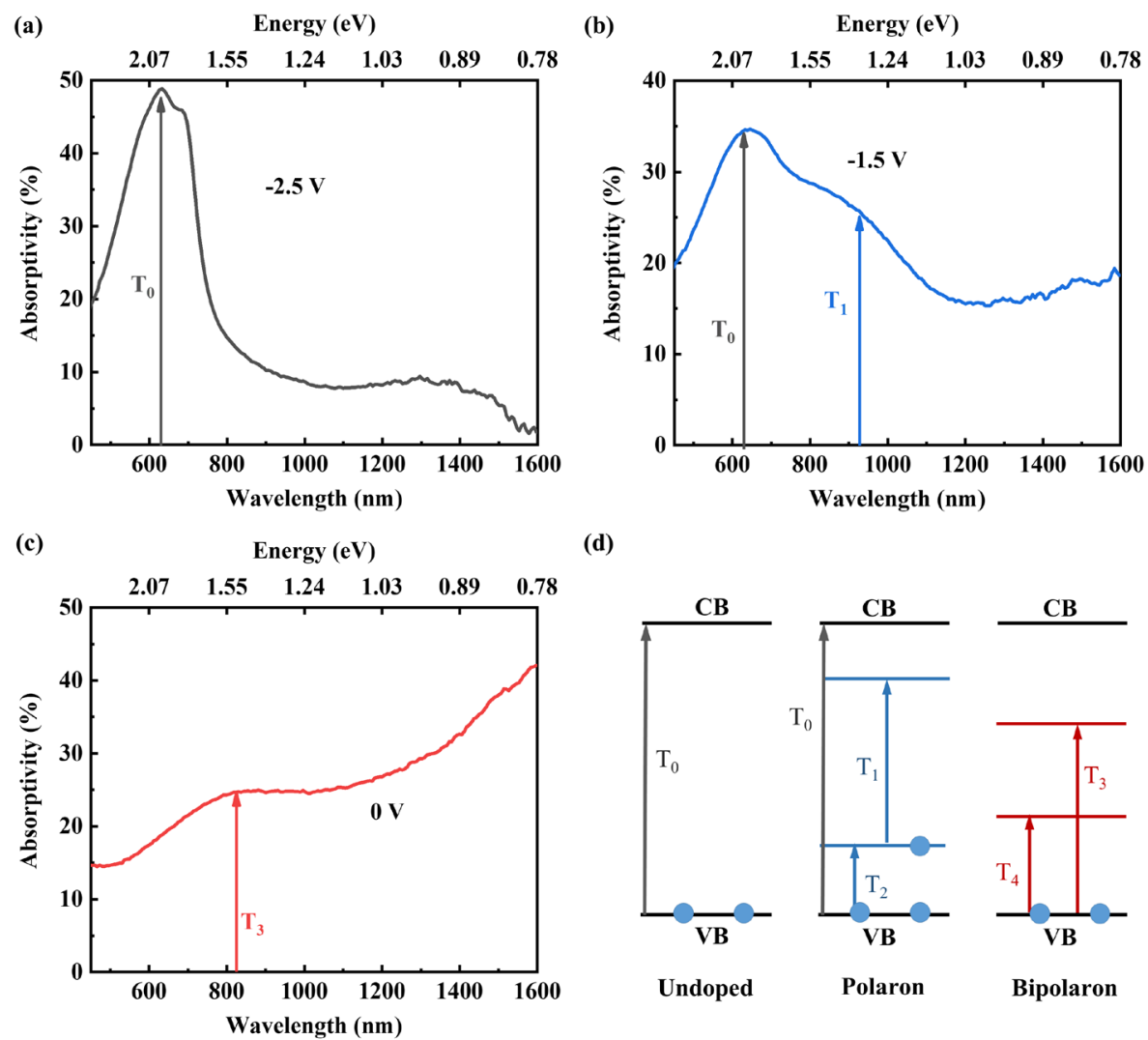

(d)

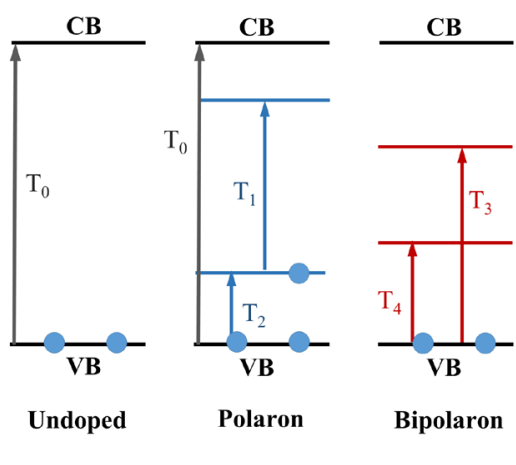

Figure 4. Absorption spectra corresponding to different doping levels of PEDOT: (a) undoped $(-2.5 \mathrm{~V}),(\mathrm{b})$ moderately doped $(-1.5 \mathrm{~V})$, and (c) heavily doped $(0 \mathrm{~V})$. (d) A proposed schematic of energy level of PEDOT for different doping levels.

The band structure variation during electrochemical doping can be derived from steady-state absorptance spectra corresponding to different doping states. Figure S7a and S7b in the SI show the Vis-NIR absorption spectra of the PEDOT:PSS film with forward and backward $\mathrm{V}_{\mathrm{A}}$ modulation, respectively. Similar to those suggested by Raman spectra, the absorption spectra show prominently hysteretic characteristics. Three typical absorption spectra are shown in panel a, b, and c of Figure 4, and a proposed schematic of energy level is drawn as Figure $4 \mathrm{~d}$ corresponding to each doping state. With a $\mathrm{V}_{\mathrm{A}}$ of $-2.5 \mathrm{~V}$, the PEDOT:PSS show a peak near $\sim 2 \mathrm{eV}$ (Figure $4 \mathrm{a}$ ) that can be ascribed to the $\pi$ - $\pi^{*}$ electron transition $\left(\mathrm{T}_{0}\right)$ of the undoped sample. The corresponding energy is referenced as the bandgap energy $\left(\mathrm{E}_{\mathrm{g}}\right)$ of PEDOT. ${ }^{47}$ With $\mathrm{V}_{\mathrm{A}}$ increasing, transition in addition to $\pi$ - $\pi^{*}$ electron transition can be found because of 
the presence of polaron or bipolaron levels located in the bandgap, and the absorption associated with $\pi-\pi^{*}$ electron transition decreases. ${ }^{47}$ With a $\mathrm{V}_{\mathrm{A}}$ of $-1.5 \mathrm{~V}$, the absorption spectrum of PEDOT:PSS shows an apparent shoulders centered at $1.33 \mathrm{eV}$ (Figure 4b). This transition is denoted as $\mathrm{T}_{1}$, which can be associated with the transition of the first polaron level to the second polaron level because the first polaron level is semi-filled. ${ }^{48}$ Meanwhile, the electron initially located at the valance band can also be excited to the first polaron level. This transition is denoted as $\mathrm{T}_{2}(<0.5 \mathrm{eV}),{ }^{49,50}$ while it cannot be detected in our experiment due to the wavelength range of our instrument. With the $\mathrm{V}_{\mathrm{A}}$ further increasing, the polaron levels are transformed to the bipolaron states in heavily doped PEDOT. ${ }^{51}$ For a heavily doped sample which exhibits two bipolaron states, the only excitation from valence band to the second bipolaron level can be detected $\left(\mathrm{T}_{3}, 1.44 \mathrm{eV}\right)$, and transition between two bipolaron states is absent because the first bipolaron state is nearly empty. ${ }^{48}$ The transition of valance band electron to the first bipolaron level is denoted as $\mathrm{T}_{4}$, which was below $0.5 \mathrm{eV}$ and cannot be detected here. ${ }^{49,50}$ It is worth noting that the $\mathrm{V}_{\mathrm{A}}$ range of the oxidation/reduction of PEDOT:PSS suggested by UV-Vis experiment to some extend deviates from that shown in Figure 3d. The deviation is associated with the fact that in UV-Vis spectroscopy the voltage was applied to the sample for a sufficiently long time, while in CV experiment the variation of $\mathrm{V}_{\mathrm{A}}$ is much faster. 


\subsection{Mechanism of the Electrical Tuning of Optical Nonlinearity}
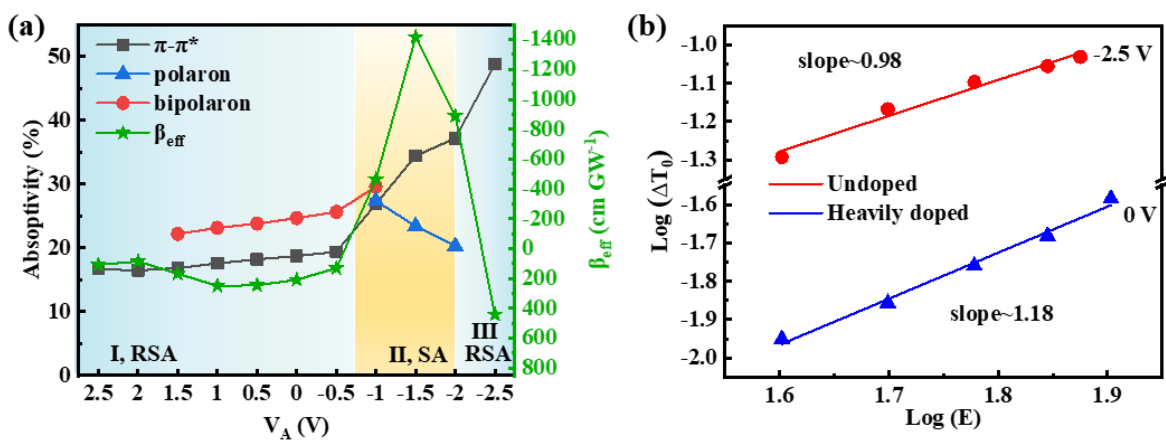

Figure 5. (a) The $\pi-\pi^{*}$, polaron, and the bipolaron absorptivity of the PEDOT:PSS film, as well as the NLO absorption coefficient of PEDOT:PSS film with forward $V_{A}$ modulation; (b) The relationship of $\Delta \mathrm{T}_{0}$ and laser pulse energy (E) in log-log scale for the PEDOT:PSS film with the $\mathrm{V}_{\mathrm{A}}$ of $-2.5 \mathrm{~V}$ and $0 \mathrm{~V}$, respectively. The data are recorded in the forward $\mathrm{V}_{\mathrm{A}}$ modulation.

In order to correlate the NLO response of the PEDOT:PSS film with different voltages to the electronic state of the PEDOT, the linear absorptivity at the wavelengths corresponding to $\pi-\pi^{*}$ transition, polaron-related transition, and bipolaron-related transition are shown together with $\beta_{\text {eff }}$ as a function of $V_{A}$ in forward $V_{A}$ modulation (Figure 5a). In region $\mathrm{I}\left(\mathrm{V}_{\mathrm{A}}\right.$ in the range $2.5 \mathrm{~V}$ to $\left.-0.5 \mathrm{~V}\right)$ the PEDOT shows an RSA response, and in this case, the linear absorption comes primarily from $\mathrm{T}_{3}$ associated with the transition of $\pi$ electrons to the second bipolaron state/band. With the $\mathrm{V}_{\mathrm{A}}$ in the range -1.0 and $-2.0 \mathrm{~V}$ (region II), strong SA can be detected. In this region the contribution of polaron transition becomes prominent. In region III $\left(a \mathrm{~V}_{\mathrm{A}}\right.$ of $\left.-2.5 \mathrm{~V}\right)$, the sample shows again RSA response. Only $\pi-\pi^{*}$ transition can be found in the absorption spectra. The relationship between NLO response and the absorptivity at the wavelengths corresponding to $\pi-\pi^{*}$ transition, polaron-related transition, and bipolaron-related transition in backward $\mathrm{V}_{\mathrm{A}}$ modulation (Figure S8 in the $\mathrm{SI}$ ) resembles 
well that in forward modulation. The NLO response in region I and III confirms that $\pi$ $\pi^{*}$ transition and bipolaron-related transition would be responsible for the RSA, while SA can be ascribed to polaron-related transition. The compromise between polaronrelated transition and $\pi-\pi^{*}$ transition at the boundary between region II and region III and that between polaron-related transition and bipolaron-related transition at the boundary between region I and region II result in the smallest $\beta_{\text {eff }}$ (strongest SA) in the center of region II.

In the $\mathrm{V}_{\mathrm{A}}$ region where the PEDOT shows $\mathrm{SA}$ response, the PEDOT exhibits intensive linear absorption at the wavelength of NLO measurement. In general, SA could arise from Pauli blocking with band filling and/or ground state depletion, ${ }^{52}$ or the red shift of absorption peak associated with electron temperature raising in the sample because of strong plasmon resonance absorption. ${ }^{31}$ Here the Pauli blocking would be the major origin of $\mathrm{SA}$, because the linear absorption at $800 \mathrm{~nm}$ in this $\mathrm{V}_{\mathrm{A}}$ region has been associated with the transition from the lower polaron level to higher polaron level ( $\mathrm{T}_{1}$ in Figure $\left.4 \mathrm{~d}\right)$. For moderately doped PEDOT, the density of polaron states is low, and the lower polaron level is semi-filled. The excitation will heavily deplete the electrons in the lower polaron level, and the band fill effect of the higher polaron state is prominent. These two effects both result in Pauli blocking and therefore SA. SA associated with ground-state depletion of in-gap states is in good accordance with those reported in $\mathrm{Fe}_{2} \mathrm{O}_{3}$ and $\mathrm{WO}_{3} .{ }^{53,54}$

To confirm that the RSA in regions I and III are the third-order nonlinearity, the Zscan experiments were carried out with the laser excitation with different pulse energies. 
The normalized transmittance changes $\left(\Delta \mathrm{T}_{0}\right.$, the depth of the valley of $\mathrm{T}_{\mathrm{NL}}(\mathrm{Z})$ curves) are plotted as a function of laser pulse energy (E) in the log-log scale (Figure 5b). Linear fitting results in a slope of 0.98 for a $\mathrm{V}_{\mathrm{A}}$ of $-2.5 \mathrm{~V}$ and 1.14 for a $\mathrm{V}_{\mathrm{A}}$ of $0 \mathrm{~V}$, indicating that the nominal TPA occurs with these $\mathrm{V}_{\mathrm{A}}$ and the responses indeed be the third-order optical nonlinearity. ${ }^{55,56}$ For the $\mathrm{V}_{\mathrm{A}}$ of $-2.5 \mathrm{~V}, \pi-\pi^{*}$ transition is dominant, and the $\mathrm{E}_{\mathrm{g}}$ satisfies the relationship $h v<\mathrm{E}_{\mathrm{g}}<2 h v$, where $h$ is Planck constant and $v$ is the frequency of the laser. Therefore, the RSA in the region III can be associated with a TPA process. The PEDOT:PSS film also shows an RSA response under $\mathrm{V}_{\mathrm{A}}$ where the bipolaronrelated excitation prevails (Region I). In this case, the energy of the incident photon $(h v)$ is sufficiently large to excite $\pi$ electrons in valence band (VB) to the second bipolaron level/band $\left(\mathrm{T}_{3}\right.$ in Figure $\left.4 \mathrm{~d}\right)$, the absorption cross-section of second bipolaron level/band is larger than that of valence band so that the electron excited from VB to bipolaron level or band can be further excited to the conduction band (CB). Namely, ESA is responsible for RSA for heavily doped PEDOT.

(a)

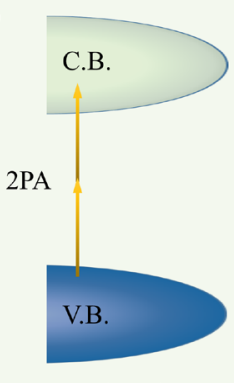

Undoped

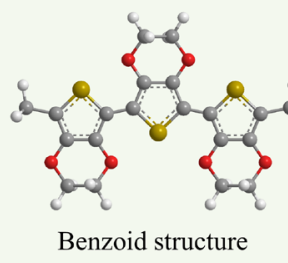

Benzoid structure (b)

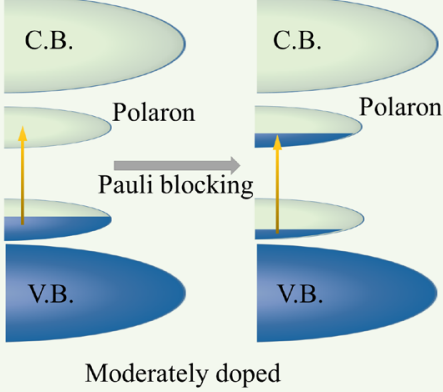

Moderately doped

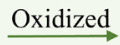

$\stackrel{4}{\text { Reduced }}$

Reduced

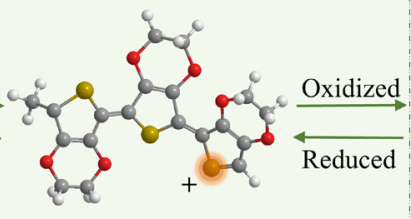

Quinoid structure (c)

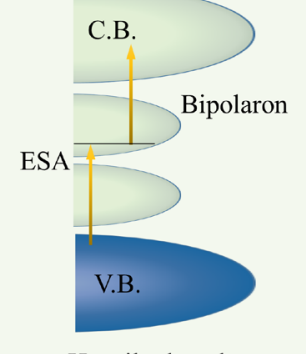

Heavily doped

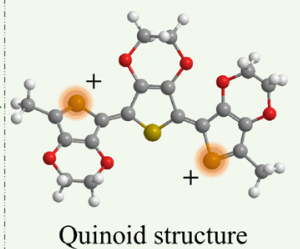

Quinoid structure

Figure 6. Proposed mechanism of the modulation of nonlinear absorption with voltage. Schematic of the density of state and molecular structure of PEDOT under different 
doping states: (a) undoped, (b) moderately doped, and (c) heavily doped.

With the nature of the electron state and the origin of the NLO process revealed, the mechanism responsible for the $\mathrm{V}_{\mathrm{A}}$-related NLO response of the PEDOT:PSS film is proposed (Figure 6). With a $\mathrm{V}_{\mathrm{A}}$ of $-2.5 \mathrm{~V}$, the PEDOT presents in the undoped state, and exhibits benzoid structure. In this case, no in-gap state can be formed, and the PEDOT shows an RSA response that can be associated with the TPA mechanism (Figure 6a). When the PEDOT is gradually oxide, it transforms into quinoid structure, and two in-gap polaron levels are formed. The lower polaron level is half-filled so that the incident laser can induce a transition from the lower polaron level to the higher polaron level. In this case, the Pauli blocking effect is prominent and the PEDOT shows a SA response (Figure 6b). With the PEDOT was further oxidized, the PEDOT exhibits abundant bipolaron states, and the bipolaron states are nearly empty. ${ }^{48}$ In this case, the higher bipolaron level/band acts as an effective intermediate state for ESA and results in RSA (Figure 6c).

Although they are all located in the bandgap of PEDOT, polaron and bipolaron level/band result in different NLO responses, with SA for polaron-related NLO and RSA for bipolaron-related NLO. The difference arises from the detailed band structure and occupation feature of the polaron and bipolaron level. The number of polaron levels is much less than that of the bipolaron level, and the polaron level is half-filled while the bipolaron level is nearly empty. ${ }^{46}$ Consequently, the ground state depletion and/or band filling are prominent for the transition between two polaron levels because the number of occupied polaron states is relatively small. In contrast, the amount of empty 
bipolaron levels is sufficiently large so that the band-filling effect is indistinct, and these empty bipolaron levels act as intermediate states that facilitate the RSA. Briefly, the amount and filling feature of in-gap states influence heavily NLO response.

\section{Conclusions}

To be concluded, the third-order nonlinear absorption of the PEDOT:PSS film was electrochemically modulated. The PEDOT:PSS film exhibits RSA or SA depending on the external bias. The corresponding $\beta_{\text {eff }}$ can be tuned in a range -1590 to $518 \mathrm{~cm} \mathrm{GW}^{-}$

1. The doping level and energy structure of PEDOT vary with external voltage. The RSA is induced by TPA in the undoped sample, and ESA in heavily doped PEDOT, while the Pauli blocking in moderately doped PEDOT results in SA. The modulation of $\beta_{\text {eff }}$ is reversible. Our results provide a facile and applicable approach for the modulation of nonparametric optical nonlinearity.

\section{Acknowledgements}

This research was financially supported by the National Natural Science Foundation of China $(51772214,51432006)$, the Ministry of Science and Technology of China (2011DFG52970), the Ministry of Education of China (IRT14R23), 111 Project (B13025), Jiangsu Province (2011-XCL-019 and 2013-479), and the Innovation Program of Shanghai Municipal Education Commission. M.G.H. and C.Z. thank the Australian Research Council for support (DP170100411). 


\section{Experimental}

Fabrication of PEDOT:PSS film: FTO substrates (Nippon Sheet Glass Co. Ltd.) were cleaned through successive ultrasonic treatment with detergent, acetone, ethanol, and deionized water each for $15 \mathrm{~min}$. The clean FTO substrate was pretreated with UVozone for $10 \mathrm{~min}$. The PEDOT:PSS films were deposited onto the FTO substrate by spin-coating (2300 rpm, 30 s) a PEDOT:PSS aqueous dispersion (Clevios PH1000), and the sample was then baked at $120{ }^{\circ} \mathrm{C}$ for 15 min under ambient atmosphere.

Assembly of device: The PEDOT:PSS film loaded onto the FTO substrate serves as a working electrode, and another FTO glass was mounted on the top of it using a Surlyn ${ }^{\circledR}$ membrane (DuPont). After that, the cavity was injected with ionic liquid (IL) of EMIM$\mathrm{BF}_{4}$ (Adamas Reagent Ltd.) and then sealed with UV-curing adhesive. The schematic structure of the PEDOT:PSS device is shown in Figure $1 \mathrm{~b}$.

Characterization: AFM experiment was carried out on a Bruker Dimension ICON. A SU8010 was utilized in SEM experiment. Raman spectra measurements were carried out with a inVia ${ }^{\mathrm{TM}}$ confocal Raman microscope (Renishaw, UK). The excitation source is $532 \mathrm{~nm}$ laser with the excitation power of $0.5 \mathrm{~mW}$ for in-situ Raman spectra.

Optical spectra and Z-scan measurement: Vis-NIR spectro-electrochemical experiments were performed using two fiber spectrometers (EK2000-Pro and EN1700, Choptics Instruments) and an electrochemistry working station (CHI760, CH Instruments). A bare device (a device assembled by two bare FTO substrates and injected with IL) was used as the back-bottom deduction to ensure that the measured data only reflect the optical absorption of PEDOT: PSS film. Single-beam nonlinear 
transmittance measurements were performed on the sample through a Z-scan system. The normalized transmission of the sample was measured with a laser beam ( $34 \mathrm{fs}, 800$ $\mathrm{nm})$ with a spot diameter of $\sim 36 \mu \mathrm{m}$.

The effective nonlinear absorption coefficient $\left(\beta_{\text {eff }}\right)$ was calculated by using the following expression: ${ }^{57}$

$$
\begin{gathered}
T_{N L}(Z)=\sum_{m=0}^{\infty} \frac{\left[-q_{0}(Z)\right]^{m}}{(m+1)^{\frac{3}{2}}} \\
q_{0}(Z)=\frac{\beta_{e f f I_{0} L_{e f f}}^{1+\left(\frac{Z}{z_{0}}\right)^{2}}}{\alpha_{0}} \\
L_{e f f}=\frac{1-e^{-\alpha_{0} L}}{\alpha_{0}}
\end{gathered}
$$

where $\mathrm{Z}$ is the sample coordinate, $\mathrm{I}_{0}$ is the on-axis irradiance at focus, $Z_{0}$ is the diffraction length of the laser beam, $\alpha_{0}$ is the linear absorption coefficient of the sample, $\mathrm{L}$ is the thickness.

\section{References}

(1) Eaton, D. F.; Meredith, G. R.; Miller, J. S. Molecular nonlinear optical materialspotential applications. Adv. Mater. 1991, 3, 564-565.

(2) Li, D. Q.; Ratner, M. A.; Marks, T. J. Molecular and Macromolecular Nonlinear Optical Materials. Probing Architecture/Electronic Structure/Frequency Doubling Relationships via an SCF-LCAO MECI $\pi$ Electron Formalism. J. Am. Chem. Soc. 1988, 110, 1707-1715.

(3) Terhune, R. W.; Maker, P. D.; Savage, C. M. Optical Harmonic Generation in Calcite. Phys. Rev. Lett. 1962, 8, 10. 
(4) Cai, W.; Vasudev, A. P.; Brongersma, M. L. Electrically Controlled Nonlinear Generation of Light with Plasmonics. Science 2011, 333, 1720-1723.

(5) Kang, L.; Cui, Y.; Lan, S.; Rodrigues, S. P.; Brongersma, M. L.; Cai, W. Electrifying photonic metamaterials for tunable nonlinear optics. Nat. Commun. 2014, 5, 4680.

(6) Lan, S.; Rodrigues, S.; Cui, Y.; Kang, L.; Cai, W. Electrically Tunable Harmonic Generation of Light from Plasmonic Structures in Electrolytes. Nano Lett. 2016, $16,5074-5079$.

(7) Ding, W.; Zhou, L.; Chou, S. Y. Enhancement and electric charge-assisted tuning of nonlinear light generation in bipolar plasmonics. Nano Lett. 2014, 14, 2822-2830.

(8) Cox, J. D.; Javier García de Abajo, F. Electrically tunable nonlinear plasmonics in graphene nanoislands. Nat. Commun. 2014, 5, 5725.

(9) Lee, K. T.; Taghinejad, M.; Yan, J.; Kim, A. S.; Raju, L.; Brown, D. K.; Cai, W. Electrically Biased Silicon Metasurfaces with Magnetic Mie Resonance for Tunable Harmonic Generation of Light. ACS Photonics 2019, 6, 2663-2670.

(10) Seyler, K. L.; Schaibley, J. R.; Gong, P.; Rivera, P.; Jones, A. M.; Wu, S.; Yan, J.; Mandrus, D. G.; Yao, W.; Xu, X. Electrical control of second-harmonic generation in a $\mathrm{WSe}_{2}$ monolayer transistor. Nat. Nanotechnol. 2015, 10, 407-411.

(11) Wang, Y.; Xiao, J.; Zhu, H.; Li, Y.; Alsaid, Y.; Fong, K. Y.; Zhou, Y.; Wang, S.; Shi, W. Structural phase transition in monolayer $\mathrm{MoTe}_{2}$ driven by electrostatic doping. Nature 2017, 550, 487-491.

(12) Klein, J.; Wierzbowski, J.; Steinhoff, A.; Florian, M.; Rösner, M.; Heimbach, F.; Müller, K.; Jahnke, F.; Wehling, T. O.; Finley, J. J. Electric-Field Switchable 
Second-Harmonic Generation in Bilayer $\mathrm{MoS}_{2}$ by Inversion Symmetry Breaking. Nano Lett. 2017, 17, 392-398.

(13) Soavi, G.; Wang, G.; Rostami, H.; Purdie, D. G.; Fazio, D. d.; Ma, T.; Luo, B.; Wang, J.; Ott, A. K.; Yoon, D. Broadband, electrically tunable third-harmonic generation in graphene. Nat. Nanotechnol. 2018, 13, 583-588.

(14) Ren, M. L.; Berger, J. S.; Liu, W.; Liu, G.; Agarwal, R. Strong modulation of second-harmonic generation with very large contrast in semiconducting $\mathrm{CdS}$ via high-field domain. Nat. Commun. 2018, 9, 186.

(15) Chen, S.; Li, K. F.; Li, G.; Cheah, K. W.; Zhang, S. Gigantic electric-field-induced second harmonic generation from an organic conjugated polymer enhanced by a band-edge effect. Light Sci. Appl. 2019, 8, 17.

(16) Li, D.; Xiong, W.; Jiang, L.; Xiao, Z.; Lu, Y. Multimodal Nonlinear Optical Imaging of $\mathrm{MoS}_{2}$ and $\mathrm{MoS}_{2}$-Based van der Waals Heterostructures. ACS Nano 2016, 10, 3766-3775.

(17) Li, Y.; Dong, N.; Zhang, S.; Zhang, X.; Feng, Y.; Wang, K.; Zhang, L.; Wang, J. Giant two - photon absorption in monolayer $\mathrm{MoS}_{2}$. Laser Photonics Rev. 2015, 9 , $427-434$.

(18) Shcherbakov, M. R.; Vabishchevich, P.; Shorokhov, A.; Chong, K. E.; Choi, D.-Y.; Staude, I.; Miroshnichenko, A. E.; Neshev, D. N.; Fedyanin, A. A.; Kivshar, Y. S. Ultrafast all-optical switching with magnetic resonances in nonlinear dielectric nanostructures. Nano Lett. 2015, 15, 6985-6990.

(19) Chen, Y.; Jiang, G.; Chen, S.; Guo, Z.; Yu, X.; Zhao, C.; Zhang, H.; Bao, Q.; Wen, 
S.; Tang, D. Mechanically exfoliated black phosphorus as a new saturable absorber for both Q-switching and mode-locking laser operation. Opt. Exp. 2015, 23, $12823-12833$.

(20) Cifuentes, M. P.; Powell, C. E.; Morrall, J. P.; McDonagh, A. M.; Lucas, N. T.; Humphrey, M. G.; Samoc, M.; Houbrechts, S.; Asselberghs, I.; Clays, K. Electrochemical, spectroelectrochemical, and molecular quadratic and cubic Nonlinear optical properties of alkynylruthenium dendrimers. J. Am. Chem. Soc. 2006, 128, 10819-10832.

(21) Powell, C. E.; Cifuentes, M. P.; Morrall, J. P.; Stranger, R.; Humphrey, M. G.; Samoc, M.; Luther-Davies, B.; Heath, G. A. Organometallic complexes for nonlinear optics. 30. Electrochromic linear and nonlinear optical properties of alkynylbis(diphosphine)ruthenium complexes. J. Am. Chem. Soc. 2003, 125, 602610.

(22) Powell, C. E.; Humphrey, M. G.; Cifuentes, M. P.; Morrall, J. P.; Samoc, M.; Luther-Davies, B. Organometallic complexes for nonlinear optics. 33. Electrochemical switching of the third-order nonlinearity observed by simultaneous femtosecond degenerate four-wave mixing and pump-probe measurements. J. Phys. Chem. A 2003, 107, 11264-11266.

(23) Cifuentes, M. P.; Powell, C. E.; Humphrey, M. G.; Heath, G. A.; Samoc, M.; Luther-Davies, B. Organometallic complexes for nonlinear optics. 24. Reversible electrochemical switching of nonlinear absorption. J. Phys. Chem. A 2001, 105, $9625-9627$. 
(24) Feng, Q.; Li, Y.; Shi, G.; Wang, L.; Zhang, W.; Li, K.; Hou, H.; Song, Y. A photocontrollable third-order nonlinear optical (NLO) switch based on a rhodamine B salicylaldehyde hydrazone metal complex. J. Mater. Chem. C 2016, 4, 8552-8558.

(25) Malti, A.; Edberg, J.; Granberg, H.; Khan, Z. U.; Andreasen, J. W.; Liu, X.; Zhao, D.; Zhang, H.; Yao, Y.; Brill, J. W. An Organic Mixed Ion-Electron Conductor for Power Electronics. Adv. Sci. 2016, 3, 1500305.

(26) Mitraka, E.; Jafari, M. J.; Vagin, M.; Liu, X.; Fahlman, M.; Ederth, T.; Berggren, M.; Jonsson, M. P.; Crispin, X. Oxygen-induced doping on reduced PEDOT. J. Mater. Chem. A 2017, 5, 4404-4412.

(27) Asplund, M.; Thaning, E.; Lundberg, J.; Sandberg-Nordqvist, A. C.; Kostyszyn, B.; Inganäs, O.; Holst, H. V. Toxicity evaluation of PEDOT/biomolecular composites intended for neural communication electrodes. Biomed. Mater. 2009, 4, 45009.

(28) Robinson, L.; Isaksson, J.; Robinson, N. D.; Berggren, M. Electrochemical control of surface wettability of poly(3-alkylthiophenes). Surf. Sci. 2006, 600, 148-152.

(29) Backlund, F. G.; Elfwing, A.; Musumeci, C.; Ajjan, F.; Babenko, V.; Dzwolak, W.; Solin, N.; Inganas, O. Conducting microhelices from self-assembly of protein fibrils. Soft Matter 2017, 13, 4412-4417.

(30) Groenendaal, B. L.; Jonas, F.; Freitag, D.; Pielartzik, H.; Reynolds, JR. Poly(3,4ethylenedioxythiophene) and its derivatives: Past, present, and future. Adv. Mater. 2000, 12, 481-494.

(31) Alam, M. Z.; Leon, I. de; Boyd, R. W. Large optical nonlinearity of indium tin 
oxide in its epsilon-near-zero region. Science 2016, 352, 795-797.

(32) Guo, Q.; Yao, Y.; Luo, Z. C.; Qin, Z.; Xie, G.; Liu, M.; Kang, J.; Zhang, S.; Bi, G.; Liu, X. Universal Near-Infrared and Mid-Infrared Optical Modulation for Ultrafast Pulse Generation Enabled by Colloidal Plasmonic Semiconductor Nanocrystals. ACS Nano 2016, 10, 9463-9469.

(33) Guo, Q.; Cui, Y.; Yao, Y.; Ye, Y.; Yang, Y.; Liu, X.; Zhang, S.; Liu, X.; Qiu, J.; Hosono, H. A Solution-Processed Ultrafast Optical Switch Based on a Nanostructured Epsilon-Near-Zero Medium. Adv. Mater. 2017, 29, 1700754.

(34) Tian, X.; Luo, H.; Wei, R.; Zhu, C.; Guo, Q.; Yang, D.; Wang, F.; Li, J.; Qiu, J. An Ultrabroadband Mid-Infrared Pulsed Optical Switch Employing SolutionProcessed Bismuth Oxyselenide. Adv. Mater. 2018, 30, 1801021.

(35) Zhang, X.; Liu, S.; Tan, D.; Xian, Y.; Zhang, D.; Zhang, Z.; Liu, Y.; Liu, X.; Qiu, J. Photochemically Derived Plasmonic Semiconductor Nanocrystals as an Optical Switch for Ultrafast Photonics. Chem. Mater. 2020, 32, 3180-3187.

(36) Zhang, S.; Dong, N.; McEvoy, N.; O'Brien, M.; Winters, S.; Berner, N. C.; Yim, C.; Li, Y.; Zhang, X.; Chen, Z. Direct Observation of Degenerate Two-Photon Absorption and Its Saturation in $\mathrm{WS}_{2}$ and $\mathrm{MoS}_{2}$ Monolayer and Few-Layer Films. ACS Nano 2015, 9, 7142-7150.

(37) Wang, K.; Szydłowska, B. M.; Wang, G.; Zhang, X.; Wang, J. J.; Magan, J. J.; Zhang, L.; Coleman, J. N.; Wang, J.; Blau, W. J. Ultrafast Nonlinear Excitation Dynamics of Black Phosphorus Nanosheets from Visible to Mid-Infrared. ACS Nano 2016, 10, 6923-6932. 
(38) Wang, K.; Wang, J.; Fan, J.; Lotya, M.; O’Neill, A.; Fox, D.; Feng, Y.; Zhang, X.; Jiang, B.; Zhao, Q. Ultrafast Saturable Absorption of Two-Dimensional $\mathrm{MoS}_{2}$ Nanosheets. ACS Nano 2013, 7, 9260-9267.

(39) Kumar, S.; Anija, M.; Kamaraju, N.; Vasu, K. S.; Subrahmanyam, K. S.; Sood, A. K.; Rao, C. N. R. Femtosecond carrier dynamics and saturable absorption in graphene suspensions. Appl. Phys. Lett. 2009, 95, 191911.

(40) Elim, H. I.; Ji, W.; Ng, M. T.; Vittal, J. J. AgInSe2 nanorods: A semiconducting material for saturable absorber. Appl. Phys. Lett. 2007, 90, 33106.

(41) Elim, H. I.; Yang, J.; Lee, J. Y.; Mi, J.; Ji, W. Observation of saturable and reversesaturable absorption at longitudinal surface plasmon resonance in gold nanorods. Appl. Phys. Lett. 2006, 88, 83107.

(42) Gallagher, K. G.; Croy, J. R.; Balasubramanian, M.; Bettge, M.; Abraham, D. P.; Burrell, A. K.; Thackeray, M. M. Correlating hysteresis and voltage fade in lithiumand manganese-rich layered transition-metal oxide electrodes. Electrochem. Commun. 2013, 33, 96-98.

(43) Garreau, S.; Duvail, J. L.; Louarn, G. Spectroelectrochemical studies of poly(3,4ethylenedioxythiophene) in aqueous medium. Synth. Met. 2001, 125, 325-329.

(44) Yang, C. S.; Shang, D. S.; Liu, N.; Shi, G.; Shen, X.; Yu, R. C.; Li, Y. Q.; Sun, Y. A Synaptic Transistor based on Quasi-2D Molybdenum Oxide. Adv. Mater. 2017, 29,1700906

(45) Louarn, G.; Trznadel, M.; Buisson, J. P.; Laska, J.; Pron, A.; Lapkowski, M.; Lefrant, S. Raman Spectroscopic Studies of Regioregular Poly(3-alkylthiophenes). 
J. Phys. Chem. 1996, 100, 12532-12539.

(46) Heeger, A. J. Charge Storage in Conducting Polymers: Solitons, Polarons, and Bipolarons. Polym. J. 1985, 17, 201-208.

(47) Gueye, M. N.; Carella, A.; Faure-Vincent, J.; Demadrille, R.; Simonato, J. P. Progress in understanding structure and transport properties of PEDOT-based materials: A critical review. Prog. Mater. Sci. 2020, 108, 100616.

(48) Patil, A. O.; Heeger, A. J.; Wudl, F. Optical-Propertoes of Conducting Polymers. Chem. Rev. 1988, 88, 183-200.

(49) Bubnova, O.; Khan, Z. U.; Malti, A.; Braun, S.; Fahlman, M.; Berggren, M.; Crispin, X. Optimization of the thermoelectric figure of merit in the conducting polymer poly(3,4-ethylenedioxythiophene). Nat. Mater. 2011, 10, 429-433.

(50) Gustafsson, J. C.; Liedberg, B.; Inganfis, O. In situ spectroscopic investigations of electrochromism and ion transport in a poly (3,4-ethylenedioxythiophene) electrode in a solid state electrochemical cell. Solid State Ionics 1994, 69, 145-152.

(51) Chung, T. C.; Kaufman, J. H.; Heeger, A. J.; Wudl, F. Charge storage in doped poly(thiophene): Optical and electrochemical studies. Phys. Rev. B 1984, 30, 702.

(52) Zitter, R. N. Saturated Optocal Absorption Through Band Filling in Semiconductors. Appl. Phys. Lett. 1969, 14, 73-74.

(53) Sachs, M.; Park, J. S.; Pastor, E.; Kafizas, A.; Wilson, A. A.; Francas, L.; Gul, S.; Ling, M.; Blackman, C.; Yano, J. Effect of oxygen deficiency on the excited state kinetics of $\mathrm{WO}_{3}$ and implications for photocatalysis. Chem. Sci. 2019, 10, 56675677. 
(54) Barroso, M.; Pendlebury, S. R.; Cowan, A. J.; Durrant, J. R. Charge carrier trapping, recombination and transfer in hematite (alpha- $\mathrm{Fe}_{2} \mathrm{O}_{3}$ ) water splitting photoanodes. Chem. Sci. 2013, 4, 2724-2734.

(55) Correa, D. S.; Boni, L. de; Misoguti, L.; Cohanoschi, I.; Hernandez, F. E.; Mendonca, C. R. Z-scan theoretical analysis for three-, four- and five-photon absorption. Opt. Commun. 2007, 277, 440-445.

(56) Zhang, Q.; Liu, X.; Utama, M. I. B.; Xing, G.; Sum, T. C.; Xiong, Q. PhononAssisted Anti-Stokes Lasing in ZnTe Nanoribbons. Adv. Mater. 2016, 28, 276-283.

(57) Sheik-Bahae, M.; Said, A. A.; Wei, T. H.; Hagan, D. J.; Van Stryland, E. W. Sensitive measurement of optical nonlinearities using a single beam. IEEE $J$. Quantum Electron. 1990, 26, 760-769. 